\title{
Lessons from (2+1)-dimensional quantum gravity
}

\author{
Bernd Schroers* \\ Department of Mathematics and Maxwell Institute for Mathematical Sciences, \\ Heriot-Watt University, \\ Edinburgh EH14 4AS, UK \\ E-mail: bernd@ma.hw.ac.uk
}

Proposals that quantum gravity gives rise to non-commutative spacetime geometry and deformations of Poincaré symmetry are examined in the context of (2+1)-dimensional quantum gravity. The results are expressed in five lessons, which summarise how the gravitational constant, Planck's constant and the cosmological constant enter the non-commutative and noncocommutative structures arising in (2+1)-dimensional quantum gravity. It is emphasised that the much studied bicrossproduct $\kappa$-Poincaré algebra does not arise directly in (2+1)-dimensional quantum gravity

From Quantum to Emergent Gravity: Theory and Phenomenology

June 11-15 2007

Trieste, Italy

${ }^{*}$ Speaker. 


\section{Introduction and motivation}

A key motivation for the study of (2+1)-dimensional quantum gravity is to shed light on general and conceptual issues associated with quantising gravity [1]. The goal of this talk is to focus on two closely related issues, namely the role of non-commutative geometry and the emergence of deformed versions of special relativity in quantum gravity, and to extract lessons regarding these issues from (2+1)-dimensional quantum gravity.

In the course of the talk I need to refer to some of the technical tools which make classical and quantum gravity in $2+1$ dimensions tractable, such as the formulation as a Chern-Simons theory and techniques from the theory of quantum groups. However, I shall try to express each of the lessons in simple, physical terms. There will be a total of five lessons, and all of them involve in an essential way the physical constants which enter quantum gravity, namely the speed of light $c$, the gravitational constant $G$, the cosmological constant $\Lambda_{c}$ and Planck's constant $\hbar$. We will mostly set the speed of light to one, but exhibit the other constants explicitly. A special feature of $2+1$ dimensions is that the Planck mass can be expressed in terms of $G$ only - without involving $\hbar$. The reason for this is that the dimension of $G$ in $2+1$ dimensions is that of an inverse mass. It follows that we can form two length parameters

$$
\ell_{P}=\hbar G \quad \ell_{C}=\frac{1}{\sqrt{\left|\Lambda_{c}\right|}}
$$

and one dimensionless ratio $\ell_{P} / \ell_{c}$.

The talk is based on previous work with Catherine Meusburger [2, 3, 4] and on current work with Shahn Majid [5]. I begin be reviewing basic properties of the Poincaré group and special relativity in $2+1$ dimensions. Promoting global Poincaré symmetry to a local symmetry leads to the formulation of gravity in $2+1$ dimensions as a Chern-Simons gauge theory. The cosmological constant can be introduced in this picture as a deformation parameter which changes the gauge group of the Chern-Simons theory. Quantisation deforms the gauge group to a Hopf algebra which is neither commutative nor cocommutative. However, one important lesson one learns in $2+1$ dimensions is that the so-called bicrossproduct $\kappa$-Poincare algebra, much discussed in the recent literature on deformed or doubly-special relativity (see e.g. [6] for a review), is not isomorphic to any of the Hopf algebras arising directly in the quantisation of $2+1$ gravity, contrary to what is sometimes claimed. I will conclude the talk with a careful explanation and discussion of this statement.

\section{Special relativity in $2+1$ dimensions}

\subsection{Minkowski space and it symmetries}

I denote vectors in three-dimensional Minkowski space by $x$ with coordinates $x^{a}, a=0,1,2$. The metric is $\eta_{a b}=\operatorname{diag}(+,-,-)$, so that the totally antisymmetric tensor $\varepsilon_{a b c}$ satisfies $\varepsilon^{a b c}=\varepsilon_{a b c}$. The identity component of the Lorentz group is $S O^{+}(2,1)$, which is isomorphic to $S L(2, \mathbb{R}) / \mathbb{Z}_{2} \simeq$ $S U(1,1) / \mathbb{Z}_{2}$. I denote the Lie algebra of the Lorentz group by $\mathfrak{s u}(1,1)$ in this talk; its generators are the rotation generator $J_{0}$ and the boost generators $J_{1}$ and $J_{2}$ with commutators

$$
\left[J_{a}, J_{b}\right]=\varepsilon_{a b c} J^{c} .
$$


The isometry group of Minkowski space is the Poincaré group, which plays a key role this talk. I will work with the double cover of the identity component of the Poincaré group

$$
P_{3}=S U(1,1) \ltimes \mathbb{R}^{3}
$$

with multiplication law

$$
\left(v_{1}, x_{1}\right)\left(v_{2}, x_{2}\right)=\left(v_{1} v_{2}, x_{1}+\operatorname{Ad}\left(v_{1}\right) x_{2}\right),
$$

where the notation exploits the identification of $\mathbb{R}^{3}$ with the Lie algebra $\mathfrak{s} u(1,1)$. The Lie algebra $\mathfrak{p}_{3}$ of the Poincaré group $P_{3}$ is generated by the Lorentz generators $J_{a}$ and translation generators $P_{a}$, with brackets

$$
\left[J_{a}, J_{b}\right]=\varepsilon_{a b c} J^{c}, \quad\left[J_{a}, P_{b}\right]=\varepsilon_{a b c} P^{c}, \quad\left[P_{a}, P_{b}\right]=0 .
$$

This algebra has the invariant, non-degenerate inner product

$$
\left\langle J_{a}, P_{b}\right\rangle=\eta_{a b}
$$

which will be crucial in what follows.

\subsection{Phase space of a free point particle $=($ Co $)$ adjoint orbit}

An excellent way to think about the phase space of any dynamical system is as the space of all solutions of the equations of motion. For a free relativistic particle the phase space is then the space of all timelike straight lines in Minkowski space. A given line can be parametrised by giving its direction $\hat{p}$ and one point $x$ on it. Since the points $x$ and $x+\tau \hat{p}$ lie on the same line for any $\tau \in \mathbb{R}$, it is convenient to use, instead of $x$, the vector

$$
k=x \wedge p+s \hat{p}
$$

for arbitrary but fixed $s \in \mathbb{R}$. Clearly, $k$ is invariant under $x \mapsto x+\tau \hat{p}$. A given line is then uniquely characterised by two vectors $p$ and $k$ with fixed values for $p^{2}=m^{2}$ and $p \cdot k=m s$. The space of all such lines (for given $m, s$ ) is four dimensional.

In order to derive the symplectic structure on the phase space we require an action. In the case of the free relativistic particle this action has a geometrical interpretation in terms of the coadjoint orbit method [7]. For convenience I use the inner product (2.5) to identify the dual vector space $\mathfrak{p}_{3}^{*}$ with $\mathfrak{p}_{3}$, and consider adjoint orbits instead of coadjoint orbits. In particular, I identify

$$
P_{0}^{*} \leftrightarrow J_{0}, \quad J_{0}^{*} \leftrightarrow P_{0}
$$

and consider the adjoint orbit of the Lie algebra element $m J_{0}+s P_{0}$. For $g=(v, x) \in P_{3}$ we define three-vectors $p$ and $k$ via

$$
g\left(m J_{0}+s P_{0}\right) g^{-1}=p_{a} J^{a}+k_{a} P^{a},
$$

which is equivalent to $p_{a} J^{a}=\operatorname{Ad}(v) J_{0}$ and the relation (2.6) between $x, p$ and $k$. Then the action of $a$ and spinning point particle [8] can be written as

$$
I_{\text {Point Particle }}=\int d \tau p_{a} \dot{x}^{a}+s\left\langle P_{0}, v^{-1} \dot{v}\right\rangle=\int d \tau\left\langle m J_{0}+s P_{0}, g^{-1} \dot{g}\right\rangle .
$$




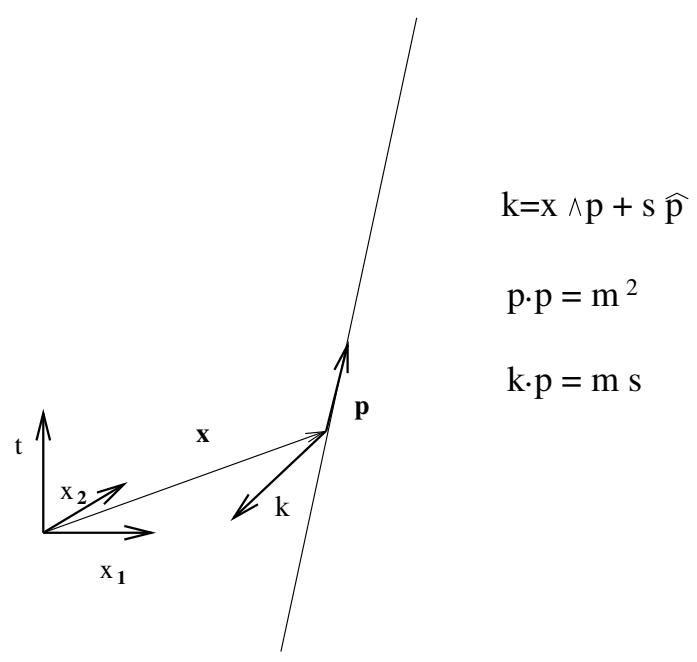

Figure 1: Parametrising the world line of a particle

The resulting Poisson brackets are the canonical Kirillov-Kostant-Souriau brackets [7] of the coordinate functions $p_{a}$ and $k_{a}$ :

$$
\left\{k_{a}, k_{b}\right\}=-\varepsilon_{a b c} k^{c}, \quad\left\{k_{a}, p_{b}\right\}=-\varepsilon_{a b c} p^{c}, \quad\left\{p_{a}, p_{b}\right\}=0 .
$$

\section{2+1 gravity as a Poincaré gauge theory}

\subsection{The Chern-Simons formulation}

The starting point for the Chern-Simons formulation of $2+1$ gravity is Cartan's trick of combining the dreibein $e_{a}$ and spin connection $\omega=\omega_{a} J^{a}$ into the one-form

$$
A=e_{a} P^{a}+\omega_{a} J^{a}
$$

with values in $\mathfrak{p}_{3}$. As observed in $[9,10]$ the Einstein-Hilbert action of $2+1$ gravity can then be written as a Chern-Simons action:

$$
I_{\text {Einstein-Hibert }}=\frac{1}{8 \pi G} \int_{M_{3}}\langle A \wedge d A\rangle+\frac{1}{3}\langle[A, A], \wedge A\rangle .
$$

Note that the definition of the action (but not of the connection) requires the inner product (2.5). The equation of motion following from (3.2) is the flatness condition for the curvature of the connection A:

$$
F_{A}=0
$$

This is equivalent to requiring the spin connection to be flat and torsion free, and hence to the Einstein equations. 


\subsection{Introducing point particles}

We consider a spacetime of topology

$$
M_{3}=S_{g n} \times \mathbb{R}
$$

where $S_{g n}$ is a surface of genus $g$ with $n$ marked points, and introduce local coordinates $x=\left(x_{1}, x_{2}\right)$ on the surface $S_{g n}$ as well as a coordinate $\tau$ for $\mathbb{R}$. Each of the marked points $S_{g n}$ is then decorated with a (co)adjoint orbit of the Poincare group which is coupled to the gauge field via minimal coupling. Concentrating on one marked point, with coordinate $x^{*}$, the coupling is

$$
I_{\text {Point Particle }}=\int d \tau\left\langle m J_{0}+s P_{0}, g^{-1}\left(\frac{d}{d \tau}+A_{\tau}\left(\tau, x^{*}\right)\right) g\right\rangle .
$$

The equation of motion is now

$$
F_{A}=-g\left(\mu J_{0}+\sigma P_{0}\right) g^{-1} d x_{1} \wedge d x_{2} \delta^{2}\left(x-x^{*}\right),
$$

with $\mu=8 \pi m G$ and $\sigma=8 \pi s G$. This forces the holonomy around a given puncture to lie in a fixed conjugacy class

$$
\mathscr{C}_{\mu \sigma}:=\left\{g e^{-\mu J_{0}-\sigma P_{0}} g^{-1} \mid g \in P_{3}\right\}
$$

\subsection{Holonomies and phase space}

In the Chern-Simons formulation, the phase space of $(2+1)$-dimensional gravity can be parameterised by holonomies around non-contractible loops on $S_{g n}$, see Fig. 2 and [2] for further details. Defining the extended phase space via

$$
\tilde{\mathscr{P}}=P_{3}^{2 g} \times \mathscr{C}_{\mu_{n} \sigma_{n}} \times \ldots \mathscr{C}_{\mu_{1} \sigma_{1}}
$$

the physical phase space is obtained as a finite quotient:

$$
\begin{aligned}
\mathscr{P}= & \left\{\left(A_{g}, B_{g}, \ldots, A_{1}, B_{1}, M_{n}, \ldots M_{1}\right) \in \tilde{\mathscr{P}} \mid\right. \\
& {\left.\left[A_{g}, B_{g}^{-1}\right] \ldots\left[A_{1}, B_{1}^{-1}\right] M_{n} \ldots M_{1}=1\right\} / \text { conjugation. } }
\end{aligned}
$$

The space $\mathscr{P}$ inherits a symplectic structure from the infinite-dimensional affine space of connections $A$, of which it is an infinite-dimensional symplectic quotient. The resulting symplectic structure on the phase space $\mathscr{P}$ (called Atiyah-Bott structure) can be described explicitly in a framework introduced by Fock and Rosly [11], and developed in [12] and [13], see also [2, 3] for its application in (2+1)-dimensional gravity. The basic idea is to work on the extended phase space $\tilde{\mathscr{P}}$, and to define a symplectic structure on it in such a way that the induced symplectic structure on the quotient (3.9) agrees with the Atiyah-Bott structure. As emphasised particularly in [12], the Fock-Rosly symplectic structure on $\tilde{\mathscr{P}}$ is isomorphic, via a "decoupling transformation", to a direct product symplectic structure consisting of building blocks associated to the Poisson-Lie structure of the gauge group (for us $P_{3}$ ), namely a copy of the so-called Heisenberg double for every handle on $S_{g n}$, and a symplectic leaf of the dual or Semenov-Tian-Shansky structure for every particle. For details regarding this structures see [14] and also [15, 16] for further background. 


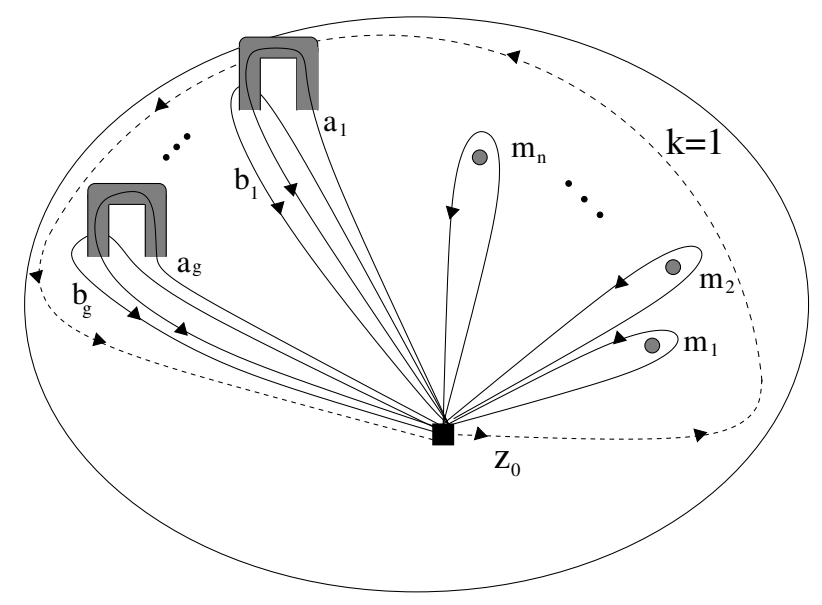

Figure 2: The generators of the fundamental group of $S_{g n}$

\section{4 $P_{3}$ as Poisson-Lie group}

A fundamental ingredient of the Fock-Rosly construction is an $r$-matrix whose defining feature is that it satisfies the classical Yang-Baxter equation, and that its symmetric part agrees (after dualising) with the inner product (2.5) used in the definition of the Chern-Simons action. It is easy to check that the r-matrix

$$
r=8 \pi G P_{a} \otimes J^{a} \in \mathfrak{p}_{3} \otimes \mathfrak{p}_{3}
$$

satisfies these requirements. Given (3.10), the group $P_{3}$ can be equipped with the Sklyanin bracket, thus turning it into a Poisson-Lie group. The bracket takes the following form in terms of the parametrisation $(v, x) \in P_{3}$ :

$$
\left\{x_{a}, x_{b}\right\}=8 \pi G \varepsilon_{a b c} x^{c}, \quad\left\{x_{a}, f(v)\right\}=\{f(v), g(v)\}=0 .
$$

As mentioned above, it is not the Sklyanin bracket itself which enters the symplectic structure of the phase space but the associated Heisenberg double and dual Poisson structures. We focus on the latter here, and note that, as a group, the dual Poisson-Lie group of $P_{3}$ is

$$
P_{3}^{*}=S U(1,1) \times \mathbb{R}^{3} .
$$

To write down the Poisson structure of $P_{3}^{*}$ explicitly, we write elements as $(u,-8 \pi G j)$, with

$$
u=\exp \left(-8 \pi G p_{a} J_{a}\right) .
$$

Then one finds the following brackets of coordinate functions:

$$
\left\{j_{a}, j_{b}\right\}=-\varepsilon_{a b c} j^{c}, \quad\left\{j_{a}, p_{b}\right\}=-\varepsilon_{a b c} p^{c}, \quad\left\{p_{a}, p_{b}\right\}=0 .
$$

The Poisson manifold $P_{3}^{*}$ is a non-linear or deformed version of the linear Poisson manifold $\mathfrak{p}_{3}^{*}$. The brackets (3.14) are precisely the same brackets as those of the coordinate functions on $\mathfrak{p}_{3}^{*}$ (2.10). However, it is important to keep in mind that for $P_{3}^{*}$, the coordinates $p_{a}$ are functions on the non-linear space $S U(1,1)$, whereas for $\mathfrak{p}_{3}^{*}$ they are functions on a linear space. 


\subsection{Conjugacy class as particle phase space}

One of the results from the theory of Poisson-Lie groups which fits very beautifully into the current story is that the symplectic leaves of $P_{3}^{*}$ are conjugacy classes in $P_{3}$. We saw earlier that holonomies around a given puncture are forced to lie in a fixed conjugacy class $\mathscr{C}_{\mu \sigma}$ of $P_{3}$, labelled by the mass and spin of the particle associated with the puncture. As shown in [12], the induced symplectic structure on those conjugacy classes is precisely that of the dual Poisson-Lie group of the gauge group, in our case $P_{3}^{*}$. The map between the conjugacy class in $P_{3}$ and the dual group $P_{3}^{*}$ is explicitly given by

$$
(v, x) e^{-\mu J_{0}-\sigma P_{0}}(v, x)^{-1}=(u,-8 \pi G \operatorname{Ad}(u) j) \mapsto(u,-8 \pi G j),
$$

with the brackets between the coordinates $j^{a}$ and $p^{a}$ as in (3.14). On the basis of those brackets we interpret $j$ as the "angular momentum" associated to the particle, and the element $u$ as a "group-valued momentum". We thus arrive at the following formulae for angular momentum and momentum in terms of the Poincaré element $(v, x)$ :

$$
\begin{aligned}
u & =v e^{-\mu J_{0}} v^{-1}=e^{-8 \pi G p_{a} J^{a}} \\
j & =\left(1-\operatorname{Ad}\left(u^{-1}\right)\right) x+s \hat{p}_{a} P_{a}=\left[x, p_{a} J^{a}\right]+s \hat{p}_{a} P_{a}+\mathscr{O}\left(p^{2}\right) .
\end{aligned}
$$

The last line shows that the formula for $j$ can be viewed as a deformed version of the relation (2.6) for a free relativistic particle. Following this analogy we think of $x^{a}$ as position coordinates. There is an important connection between the brackets (3.11) of the position coordinates and the brackets (3.14) of momentum and angular momentum: the conjugation action of $(v, x)$ on $(u,-8 \pi G \operatorname{Ad}(u) j)$ is a Poisson action only if we take into account the non-trivial Poisson brackets of the position coordinates $x^{a}$ given in (3.11). We thus arrive at

\section{LESSON 1: particle phase space in $2+1$ gravity}

- Momentum space has curvature radius $\propto \frac{1}{G}$

- Position coordinates do not Poisson commute $\propto G$

- The angular momentum Poisson algebra is unchanged - but the relation between position, momentum and angular momentum is changed

\section{Introducing the cosmological constant}

\subsection{Lie groups and Lie algebras}

In $2+1$ gravity, solutions of the Einstein equations are locally isometric to a model spacetime which is determined by the signature of spacetime (Euclidean or Lorentzian) and the cosmological constant $[17,1]$. The isometry groups of these model spacetimes are therefore local isometry groups in $2+1$ gravity. In the formulation as a Chern-Simons gauge theory $[9,10]$, the local isometry groups play the role of gauge groups. We list the groups arising for different signatures and signs of the cosmological constant in Table 1. 


\begin{tabular}{|c|c|c|}
\hline $\begin{array}{c}\text { Cosmological } \\
\text { constant }\end{array}$ & Euclidean signature & Lorentzian signature \\
\hline$\Lambda_{c}=0$ & $E_{3}$ & $P_{3}$ \\
\hline$\Lambda_{c}>0$ & $S O(4) \simeq \frac{S U(2) \times S U(2)}{\mathbb{Z}_{2}}$ & $S O(3,1) \simeq S L(2, \mathbb{C}) / \mathbb{Z}_{2}$ \\
\hline$\Lambda_{c}<0$ & $S O(3,1) \simeq S L(2, \mathbb{C}) / \mathbb{Z}_{2}$ & $S O(2,2) \simeq \frac{S U(1,1) \times S U(1,1)}{\mathbb{Z}_{2}}$ \\
\hline
\end{tabular}

Table 1: Local isometry groups in $2+1$ gravity

The Lie brackets of the associated Lie algebras can be written in unified fashion by introducing

$$
\Lambda= \begin{cases}\Lambda_{c} & \text { for Euclidean signature } \\ -\Lambda_{c} & \text { for Lorentzian signature }\end{cases}
$$

They take the following form in terms of generators $J_{a}$ and $P_{a}$ adapted to the Cartan decomposition:

$$
\left[J_{a}, J_{b}\right]=\varepsilon_{a b c} J^{c}, \quad\left[J_{a}, P_{b}\right]=\varepsilon_{a b c} P^{c} \quad\left[P_{a}, P_{b}\right]=\Lambda \varepsilon_{a b c} J^{c} .
$$

The invariant pairing remains (2.5) regardless of the value of $\Lambda$. Later we will also need the Iwasawa decomposition of the Lie algebras. As explained in [4], the generators

$$
\tilde{P}_{a}=P_{a}+\varepsilon_{a b c} n^{b} J^{c}, \quad n^{2}=-\Lambda,
$$

together with $J_{a}$ provide this decomposition. In particular one has

$$
\left[\tilde{P}_{a}, \tilde{P}_{b}\right]=n_{a} \tilde{P}_{b}-n_{b} \tilde{P}_{a} .
$$

It is explained in [4] how to write down Sklyanin, dual and Heisenberg double brackets for the gauge groups listed in Table 1; as explained earlier, this amounts to a complete description of the symplectic structure on the phase space of the theory in the Fock-Rosly framework. The cosmological constant introduces curvature into the model spacetimes of $2+1$ gravity; it is therefore not surprising that momenta, which generate translations in the model spacetime, no longer Poisson commute when the cosmological constant is non-vanishing.

\section{LESSON 2: the effect of the cosmological constant}

- If $\Lambda \neq 0$ position space has curvature radius $\propto \ell_{c}$

- $\Lambda \neq 0$ momenta do not Poisson commute $\propto \frac{1}{\ell_{c}}$

- LESSON 1 still applies. 


\section{Quantisation}

\subsection{Quantisation of free point-particle (coadjoint orbit) brackets}

The quantisation of the Poisson algebra of momenta and angular momenta of a free particle (2.10) leads to the associative algebra generated by $J_{0}, J_{1}, J_{2}$ and $P_{0}, P_{1}, P_{2}$ with relations

$$
\left[J_{a}, J_{b}\right]=\hbar \varepsilon_{a b c} J^{c}, \quad\left[J_{a}, P_{b}\right]=\hbar \varepsilon_{a b c} P^{c}, \quad\left[P_{a}, P_{b}\right]=0
$$

The resulting algebra is the universal enveloping algebra $U\left(\mathfrak{p}_{3}\right)$ [18]. Alternatively, one can think of the momenta as coordinate functions $p_{a}$ on momentum space $\left(\mathbb{R}^{*}\right)^{3}$. The $\mathfrak{s} u(1,1)$ generators act on $\left(\mathbb{R}^{*}\right)^{3}$ by infinitesimal rotations or boosts, and hence on the polynomial algebra $\operatorname{Pol}\left(\left(\mathbb{R}^{*}\right)^{3}\right)$. One can view $U\left(\mathfrak{p}_{3}\right)$ therefore also as the semi-direct product of algebras

$$
U(\mathfrak{s u}(1,1)) \ltimes \operatorname{Pol}\left(\left(\mathbb{R}^{*}\right)^{3}\right) .
$$

The description of the momentum algebra as a function algebra offers certain advantages which become manifest when one writes down the coalgebra structure which turns (5.2) into a Hopf algebra. The coalgebra structure encodes how momenta and angular momenta of several particles are combined, see [19] for details on this point of view. For a free particle this is through simple addition i.e.

$$
\Delta J_{a}=J_{a} \otimes 1+1 \otimes J_{a} \quad \text { and } \quad \Delta\left(p_{a}\right)=p_{a} \otimes 1+1 \otimes p_{a} .
$$

The last formula is a special case of the following general construction. Suppose $G$ is any Lie group, and $\mathbb{C}(G)$ is the abelian algebra of complex valued functions on $G$, with pointwise multiplication ${ }^{1}$. Then we can define a coproduct via

$$
\Delta: \mathbb{C}(G) \rightarrow \mathbb{C}(G \times G), \quad \Delta f(g, h)=f(g h) .
$$

For $G=\left(\mathbb{R}^{*}\right)^{3}$ this leads to the rule (5.3) for the coordinate functions $p_{a}$.

\subsection{The Lorentz double}

It is explained in detail in [3] how the quantisation of the Poisson brackets (3.14) of the momentum and angular momentum of a gravitating particle in $2+1$ dimensions leads to the Hopf algebra

$$
D(U(\operatorname{su}(1,1)):=U(\mathfrak{s u}(1,1)) \ltimes \mathbb{C}(S U(1,1)) .
$$

This Hopf algebra is a particular example of a quantum double, and was called Lorentz double in [19]. Following our discussion of the phase of a particle in $2+1$ gravity, it is not difficult to appreciate how this algebra arises. The angular momentum algebra is unchanged compared to the free relativistic particle, but the momentum coordinates are now functions on the group manifold $S U(1,1)$ rather than the linear space $\left(\mathbb{R}^{*}\right)^{3}$. To go from (5.2) to (5.5) we simply replace $\operatorname{Pol}\left(\left(\mathbb{R}^{*}\right)^{3}\right)$

\footnotetext{
${ }^{1}$ I do not discuss analytical aspects of this algebra in the current talk, and therefore will not specify the class of functions further; however, we do require the functions to be differentiable
} 
by the function algebra $\mathbb{C}(S U(1,1))$. Since the group $G=S U(1,1)$ is non-abelian it follows immediately that the momentum addition according to the general rule (5.4) is not cocommutative (i.e. depends on the order in the tensor product). Applying the rule (5.4) to group elements parametrised as in (3.16), and expanding in powers of $G$ one computes the leading order in non-cocommutativity. In Lesson 3 we combine this result with the usual quantisation of the Poisson brackets (3.11) for the position coordinates. Note that the lack of cocommutativity is independent of $\hbar$ and therefore really a classical effect; it is merely the manifestation of the momentum space curvature in the language of the Hopf algebra (5.5).

\section{LESSON 3: quantisation with vanishing cosmological constant}

- $\left[J_{a}, J_{b}\right]=\hbar \varepsilon_{a b c} J^{c}:$ Angular momentum coordinates do not commute $\propto \hbar$

- $\Delta\left(p_{a}\right)=1 \otimes p_{a}+p_{a} \otimes 1+8 \pi G \varepsilon_{a b c} p^{b} \otimes p^{c}+\ldots:$ Momenta do not cocommute $\propto G$

- $\left[X_{a}, X_{b}\right]=8 \pi l_{P} \varepsilon_{a b c} X^{c}$ : Position coordinates do not commute $\propto l_{P}$

\subsection{Quantisation when $\Lambda \neq 0$}

The quantisation of $2+1$ gravity has been been studied in the so-called combinatorial or Hamiltonian framework for the cases $\Lambda=0$ (for both Euclidean and Lorentzian signature, see [20] and [3]) and $\Lambda<0$ [21]. Table 2 lists the quantum groups which (are believed to) play a role analogous to that of the Lorentz double in the case $\Lambda=0$ (and Lorentzian signature). For $\Lambda>0$ the quantisation along the lines described in this talk has not been carried out in detail, so the corresponding entries are conjectural. The parameter $q$ in the table is

$$
q=e^{-\hbar G \sqrt{-\Lambda}}
$$

and combines all three physical constants which enter $2+1$ dimensional quantum gravity. As usual, $D(H)$ stands for the quantum double of a Hopf algebra $H$.

\begin{tabular}{|c|c|c|}
\hline Cosmological const. & Euclidean signature & Lorentzian signature \\
\hline$\Lambda_{c}=0$ & $D(U(\operatorname{su}(2))$ & $D(U(\operatorname{su}(1,1))$ \\
\hline$\Lambda_{c}>0$ & $D\left(U_{q}(\operatorname{su}(2))\right), q$ root of unity & $D\left(U_{q}(\operatorname{su}(1,1))\right) q \in \mathbb{R}$ \\
\hline$\Lambda_{c}<0$ & $D\left(U_{q}(\operatorname{su}(2))\right), q \in \mathbb{R}$ & $D\left(U_{q}(\operatorname{su}(1,1))\right), q \in U(1)$ \\
\hline
\end{tabular}

Table 2: Quantum groups arising in 2+1 quantum gravity 
All of the quantum groups in Table 2 are non-commutative and non-cocommutative. By studying the algebra and coalgebra structure of the quantum groups in Table 2, one can extract the parameters which control the failure of commutativity and cocommutativity to leading order. The results are summarised in the table below; it also includes a row for the position algebra, which I obtained by considering the dual Hopf algebra of the momentum algebra. See [22] for a detailed discussion of the postion algebra for the quantum double of $S U(2)$.

\begin{tabular}{|c|c|c|}
\hline LESSON 4 & Commutator & Co-commutator \\
\hline Angular momentum & $\hbar$ & $\frac{G}{\ell_{c}}$ \\
\hline Momentum & $\frac{\hbar}{\ell_{c}}$ & $G$ \\
\hline Position & $\hbar G$ & $\frac{1}{\ell_{c}}$ \\
\hline
\end{tabular}

\section{The $\kappa$-Poincaré algebra}

The so-called $\kappa$-Poincaré Hopf algebra was one of the first deformations of Poincaré symmetry proposed in the literature [23, 24]. At first sight, the $\kappa$-Poincaré algebra shares certain structural features with the Lorentz double: it has a deformation parameter with the dimension of mass, it involves a curved momentum space, and it is isomorphic to the universal enveloping algebra $U\left(\mathfrak{p}_{3}\right)$ as an algebra (though not as a Hopf algebra). As we have seen, momentum space in the Lorentz double is the group manifold $S U(1,1)$, which, as a Lorentzian manifold, is isomorphic to anti-de Sitter space. In the standard version of the (2+1)-dimensional $\kappa$-Poincaré Hopf algebra, by contrast, momentum space is de Sitter space

$$
\mathrm{dS}=\left\{\left(\pi, \pi_{3}\right) \in \mathbb{R}^{4} \mid-\pi_{0}^{2}+\pi_{1}^{2}+\pi_{2}^{2}+\pi_{3}^{2}=\kappa^{2}\right\} .
$$

The group $S U(1,1)$, and hence the Lie algebra $\mathfrak{s} u(1,1)$, act on de Sitter space. One can therefore define the semidirect product of $U(\mathfrak{s} u(1,1))$ with the algebra of complex-valued functions on $d S$ :

$$
U(\mathfrak{s} u(1,1)) \ltimes \mathbb{C}(\mathrm{dS}) .
$$

However, since de Sitter space (unlike anti de Sitter space) is not a group manifold, we cannot use our standard construction (5.4) to define a coproduct.

In order to understand the construction of the coproduct we need to take a (short) detour and review the bicrossproduct construction $[25,26]$ of which the Hopf algebra structure of the $\kappa$-Poincaré algebra is a special case [24]. The starting point of the construction is the following 
factorisation of elements of the group $S L(2, \mathbb{C})$ (strictly speaking this only holds for elements which obey a certain condition, see [4] for details):

$$
g \in S L(2, \mathbb{C}) \Rightarrow g=u \cdot s=r \cdot v, \quad u, v \in S U(1,1), \quad r, s \in A N .
$$

where $A N \simeq \mathbb{R} \ltimes \mathbb{R}^{2}$ is group of matrices of form

$$
r=\left(\begin{array}{cc}
e^{-\frac{p_{0}}{\kappa}} & \frac{p_{1}}{\kappa}+i \frac{p_{2}}{\kappa} \\
0 & e^{\frac{p_{0}}{\kappa}}
\end{array}\right)
$$

Here $p_{0}, p_{1}, p_{2}$ are real parameter which we will eventually interpret as momentum coordinates; the constant $\kappa$ has the dimension of mass, and is introduced at this point for purely dimensional reasons. Next recall that (3+1)-dimensional Minkowski space can naturally be identified with the vector space of Hermitian $2 \times 2$ matrices, and that the action of $g \in S L(2, \mathbb{C})$ on Hermitian matrices $h \mapsto g h g^{\dagger}$ implements (3+1)-dimensional Lorentz transformations. The de Sitter manifold can be realised as a submanifold of the space of Hermitian $2 \times 2$ matrices via

$$
\mathrm{d} S=\left\{\pi_{0}+\pi_{1} \sigma_{1}+\pi_{2} \sigma_{2}+\pi_{3} \sigma_{3} \mid-\pi_{0}^{2}+\pi_{1}^{2}+\pi_{2}^{2}+\pi_{3}^{2}=\kappa^{2}\right\}
$$

where $\sigma_{1}, \sigma_{2}$ and $\sigma_{3}$ are the Pauli matrices. Now note that dS is the orbit of $\kappa \sigma_{3}$ under the $\operatorname{SL}(2, \mathbb{C})$ action, and that the subgroup $S U(1,1)$ of $S L(2, \mathbb{C})$ is precisely the stabiliser group of $\kappa \sigma_{3}$. Thus, provided the second factorisation in (6.3) holds, we obtain a map

$$
A N \rightarrow \mathrm{dS}, \quad r \mapsto \kappa r \sigma_{3} r^{\dagger} .
$$

In fact, as explained in [4], the image of this map is only "half" of de Sitter space. However, if we use the image of the map (6.6) instead of all of de Sitter space as momentum space we obtain a curved momentum manifold which has a group structure (that of AN). Moreover, since de Sitter space is acted on by $(2+1)$-dimensional Lorentz transformations, we have an action of infinitesimal Lorentz transformations on the functions on "half" of de Sitter space. Thus we can define

$$
P_{\kappa}=U(\mathfrak{s u}(1,1)) \triangleright \mathbb{C}(A N)
$$

which is a semi-direct product of algebras, and has a non-cocommutative momentum coproduct

$$
\Delta\left(p_{i}\right)=p_{i} \otimes 1+e^{-\frac{p_{0}}{\kappa}} \otimes p_{i}
$$

which uses the group structure of $A N$. The symbol $\triangleright$ indicates that there is a twist in the angular momentum comultiplication, but this will not concern us here.

\section{Relation with $2+1$ gravity?}

We saw that the $\kappa$-Poincare algebra is a bicrossproduct Hopf algebra; it has some structural similarities with the Lorentz double, but is not isomorphic to it. I will end this talk by sketching some observations about how these two Hopf algebras can be related by a process called semidualisation [26]. Consider two Hopf algebras which are each other's dual as Hopf algebra, e.g.

$$
U_{q}(\mathfrak{a} n) \leftrightarrow \mathbb{C}_{q}(A N) .
$$


Semidualisation can be applied to Hopf algebras that factorise, and replaces one of the factors by its dual. For example, starting with

$$
U(\mathfrak{s l}(2, \mathbb{C})) \simeq U(\mathfrak{s} u(1,1)) \bowtie U(\mathfrak{a} n),
$$

and using the classical $(q=1)$ version of (7.1) the semidualisation map is

$$
U(\mathfrak{s} l(2, \mathbb{C})) \stackrel{S}{\rightarrow} U(\mathfrak{s} u(1,1)) \triangleright \mathbb{C}(A N)=P_{\kappa}
$$

Combining the semidualisation with the quantum duality principle [27, 28]

$$
\mathbb{C}_{q}(S U(1,1)) \simeq U_{q}(\mathfrak{a} n)
$$

which holds only when $q \neq 1$, we obtain the following diagram [5]

$$
\begin{array}{cc}
U_{q}(\mathfrak{s} u(1,1)) \bowtie \mathbb{C}_{q}(S U(1,1)) & \stackrel{q \neq 1}{\simeq} U_{q}(\mathfrak{s l}(2, \mathbb{C})) \stackrel{S}{\mapsto} U_{q}(\mathfrak{s} u(1,1)) \triangleright \mathbb{C}_{q}(A N) \\
\downarrow q \rightarrow 1 & \downarrow q \rightarrow 1 \\
D(U(\operatorname{su}(1,1))) & P_{\kappa}
\end{array}
$$

Summarising the comparison between the bicrossproduct $\kappa$-Poincaré algebra and the quantum doubles arising in $2+1$ quantum gravity is the subject of the fifths and last lesson. Before coming to that summary, I should comment on the suspiciously vague word "arising" in the previous sentence.

In this talk I have explained the technical origin of the quantum groups in Table 2 in the FockRosly description of the phase space. However, the $r$-matrices used in the Fock-Rosly construction are really auxiliary objects, used to define a Poisson structure on the extended phase space (3.8); the induced Poisson structure on the physical phase space only depends on the symmetric part of these $r$-matrices. Correspondingly, the quantum groups in Table 2 are auxiliary objects in the quantisation, and not uniquely associated to the quantum theory. Fortunately, there is independent evidence that the quantum doubles of Table 2 play an essential role in $2+1$ quantum gravity, which does not make use of the Fock-Rosly construction [29]. Thus I think it is fair to say that $2+1$ quantum gravity does provide evidence for the general idea that quantum gravity leads to a deformation of Poincaré symmetry, with a deformation parameter of dimension mass; the Lorentz double provides a specific realisation of this. I should stress that this is a deformation of Hopf algebras. A meaningful discussion must take into account both the algebra and the coalgebra structure. In the standard basis for the Lorentz double, for example, the algebra remains unchanged, and all the deformation takes place in the coalgebra.

By contrast, the role of the bicrossproduct $\kappa$-Poincaré Hopf algebra $P_{\kappa}$ in $2+1$ quantum gravity remains, to my mind, unclear. It is possible to obtain the $\kappa$-Poincaré algebra in $3+1$ dimensions by a contraction procedure from $U_{q}(s o(3,2))$ in the limit $\Lambda \rightarrow 0$ [23]. This contraction procedure is sometimes interpreted as evidence for the emergence of $P_{K}$ in a low energy limit of gravity in both $2+1$ and 3+1 dimensions, see e.g. [30]. However, I am not aware of a careful version of this argument which takes into account both the algebra and the co-algebra structure, and also keeps track of the $*$-structure (the analogue of a real structure for Hopf algebras, see e.g. [26]). It is 
not sufficient to consider the algebra alone, since the bicrossproduct $\kappa$-Poincaré algebra, like the Lorentz double, is isomorphic to the Poincaré algebra as an algebra (see e.g. [31]). The $*$-structure matters because it distinguishes, for example, $\mathfrak{s u}(2)$ from $\mathfrak{s u}(1,1)$, and therefore Euclidean from Lorentzian physics. I indicated above another way of obtaining $P_{\kappa}$ by a sequence of mathematical steps from one of the quantum doubles in Table 2; interpreting these steps physically and relating them to the contraction procedure in [23] is the subject of [5]. However, at this stage the arguments for a role of $P_{K}$ in $2+1$ gravity seem far less convincing to me than those for the Lorentz double.

In relation to $3+1$ dimensions, the situation in $2+1$, as I see it, presents a dilemma. The quantum groups which arise are all quantum doubles whose construction goes back to the essentially (2+1)-dimensional pairing (2.5). Other constructions which do generalise to higher dimensions, like the bicrossproduct construction, by contrast, do not arise naturally in $2+1$ quantum gravity.

\section{LESSON 5: $\kappa$-Poincaré versus quantum doubles}

- In 2+1 gravity momentum space is either Euclidean and positively curved (three-sphere) or Lorentzian and negatively curved (anti-de Sitter). The position algebra is $\left[X_{a}, X_{b}\right]=8 \pi \ell_{P} \varepsilon_{a b c} X^{c}$.

- In the standard bicrossproduct construction of $\kappa$-Poincaré, momentum space is Lorentzian and positively curved (de Sitter). The position algebra is $\left[X_{0}, X_{i}\right]=\ell_{P} X_{i}$.

- Lorentz double and $\kappa$-Poincaré are different Hopf algebras arising as $q \rightarrow 0$ limits of semidual Hopf-algebras .

\section{References}

[1] S. Carlip, Quantum gravity in 2+1 dimensions, Cambridge University Press, Cambridge, 1998.

[2] C. Meusburger and B J. Schroers, Poisson structure and symmetry in the Chern-Simons formulation of (2+1)-dimensional gravity, Class. Quant. Grav. 20 (2003) 2193-2233.

[3] C. Meusburger and B. J. Schroers, The quantisation of Poisson structures arising in Chern-Simons theory with gauge group $G \ltimes \mathfrak{g}^{*}$, Adv. Theor. Math. Phys. 7 (2004) 1003-1043.

[4] C. Meusburger and B. J. Schroers, Quaternionic and Poisson-Lie structures in 3d gravity: the cosmological constant as deformation parameter, arXive:0708.1507.

[5] S. Majid and B. Schroers, q-deformation and semidualisation in 2+1 quantum gravity, in preparation.

[6] J. Kowalski-Glikman, Introduction to doubly special relativity, Lect. Notes Phys. 669 (2005) 131-159; also hep-th/0405273.

[7] A. A. Kirillov, Elements of the theory of representations, Grundlehren der mathematischen Wissenschaft 220, Springer Verlag, Berlin, 1976.

[8] P. de Sousa Gerbert, On spin and (quantum) gravity in 2+1 dimensions, Nuclear Physics B346 (1990) $440-472$.

[9] A. Achucarro, P. Townsend, A Chern-Simons action for three-dimensional anti-de Sitter supergravity theories, Phys. Lett. B 180 (1986) 85-100.

[10] E. Witten, 2+1 dimensional gravity as an exactly soluble system, Nucl. Phys. B 311 (1988) 46-78. 
[11] V. V. Fock and A. A. Rosly, Poisson structures on moduli of flat connections on Riemann surfaces and $r$-matrices, ITEP preprint (1992) 72-92 (see also math.QA/9802054).

[12] A. Yu. Alekseev and A. Z. Malkin, Symplectic structure of the moduli space of flat connections on a Riemann surface, Commun. Math. Phys. 169 (1995) 99-119.

[13] A. Yu. Alekseev and V. Schomerus, Representation theory of Chern-Simons observables, Duke Math. Journal 85 (1996) 447-510.

[14] A. Yu. Alekseev and A. Z. Malkin, Symplectic structures associated to Lie-Poisson groups, Commun. Math. Phys. 162 (1994) 147-73.

[15] Y. Kosmann-Schwarzbach, Lie Bialgebras, Poisson Lie groups, and Dressing Transformations, Lect. Notes Phys 638 (2004) 107-173.

[16] V. Chari and A. Pressley, A guide to quantum groups, Cambridge University Press, Cambridge, 1994.

[17] S. Deser and R. Jackiw, Three-dimensional cosmological gravity: dynamics of constant curvature, Annals Phys. 153 (1984) 405-416.

[18] J. Dixmier, Enveloping algebras, North Holland Publishing Company, Amsterdam, 1977.

[19] F. A. Bais, N. M. Muller and B. J. Schroers, Quantum group symmetry and particle scattering in (2+1)-dimensional quantum gravity, Nucl. Phys. B 640 (2002) 3-45.

[20] B. J. Schroers, Combinatorial quantisation of Euclidean gravity in three dimensions, in: N. P. Landsman, M. Pflaum, M. Schlichenmaier (Eds.), Quantization of singular symplectic quotients, Birkhäuser, Progress in Mathematics, Vol. 198, 2001, 307-328, also math.qa/0006228.

[21] E. Buffenoir, K. Noui and P. Roche, Hamiltonian quantization of Chern-Simons theory with $S L(2, \mathbb{C})$ group, Class. Quant. Grav. 19 (2002) 4953-5016.

[22] E. Batista and S. Majid, Noncommutative geometry of angular momentum space U(su(2)), J. Math. Phys. 44 (2003), 107-137.

[23] J. Lukierski, A. Nowicki, H. Ruegg and V. N. Tolstoy, q-Deformations of Poincaré algebra, Phys. Lett. B268 (1991) 331-338.

[24] S. Majid and H. Ruegg, Bicrossproduct structure of the $\kappa$-Poincaré group and non-commutative geometry, Phys. Lett. B. 334 (1994) 348.

[25] S. Majid, Physics for algebraists: Non-commutative and non-cocommutative Hopf algebras by a bicrossproduct construction, J. Algebra 130 (1990) 17-64.

[26] S. Majid, Foundations of quantum group theory, Cambridge University Press, Cambridge, 1995.

[27] V. G. Drinfeld. Quantum groups, in: A. M. Gleason (ed), Proceedings of the International Congress of Mathematicians, Berkeley 1986, 798-820, American Mathematical Society, Providence, RI.

[28] M. A. Semenov-Tian-Shansky, Poisson-Lie groups, quantum duality principle and the quantum double, Theor. Math. Phys. 93 (1992) 1292-1307.

[29] K. Noui, Three dimensional loop quantum gravity: towards a self-gravitating quantum field theory, Class. Quant. Grav. 24 (2007) 329-360.

[30] G. Amelino-Camelia, L. Smolin and A. Starodubtsev, Quantum symmetry, the cosmological constant and Planck scale phenomenology, Class. Quant. Grav. 21 (2004) 3095-3110.

[31] J. Kowalski-Glikman and S. Nowak, Doubly special relativity theories as different basis for א-Poincaré algebra, Phys. Lett. B539 (2002) 126-132,2002. 